\title{
The Application of TELES in the Enhancement of Disaster Response and Rescue Policy in Tainan Area: A Study Following 0206 Earthquake in Tainan
}

\author{
Yuan-Ming Chiu \\ Chang Jung Christian University of Business and Operations Management, Taiwan \\ Email: yangming623@gmail.com
}

How to cite this paper: Chiu, Y.-M. (2018) The Application of TELES in the Enhancement of Disaster Response and Rescue Policy in Tainan Area: A Study Following 0206 Earthquake in Tainan. Journal of Power and Energy Engineering, 6, 1-7. https://doi.org/10.4236/jpee.2018.61001

Received: September 19, 2017

Accepted: January 1, 2018

Published: January 4, 2018

Copyright $\odot 2018$ by author and Scientific Research Publishing Inc. This work is licensed under the Creative Commons Attribution-NonCommercial International License (CC BY-NC 4.0). http://creativecommons.org/licenses/by-nc/4.0/ cc) (i) (8) Open Access

\begin{abstract}
The 2016 Meinong earthquake in Kaohsiung city caused significant casualties and property damage in Tainan. This study used TELES to simulate the earthquake scenario parameters, such as PGA distribution, casualty case, liquefaction potential index and the total number of occurring fires based on the Meinong fault seismic source. Finally, according to the most damaging areas of the simulation results, we propose to strengthen the important buildings and facilities such as the reservoir area, and the emergency responsive hospitals for a better seismic risk estimation and the earthquake disaster prevention and response. According to the simulation results analysis, we found the areas with the strongest magnitude were in Meinong Dist. in Kaohsiung city. Nevertheless, the areas suffering the serious disasters were in Tainan city. Due to the serious disasters occurring in Tainan city, it is crucial to discuss how to perform anti-earthquake practices in Tainan area and how to coordinate the support from different cities and counties.
\end{abstract}

\section{Keywords}

TELES Meishan Fault, 0206 Earthquake in Tainan, Earthquake Simulation

\section{Background/Objectives and Goals}

\subsection{Background}

At 03:57 local time on 6 February, 2016, an earthquake of Richter magnitude 6.4 broke out, and it was measured 5 in intensity in Tainan. Damages and disasters were reported one after another. The worst affected was Weiguan-Jinlong com- 
plex, which collapsed at 04:03. The dispatch center of Tainan City Government Fire Bureau immediately dispatched all kinds of rescue vehicles to the scenes of all the affected areas. At 4:10, minutes after the earthquake, the mayor of Tainan City William Lai rushed to the dispatch center and ordered that an emergency response center be set up. Weiguan-Jinlong was a 16-story tall residential and commercial complex with a one-story basement. As shown in Figure 1, it consisted of nine buildings and each was marked A, B, C, D, E, F, G, H, and I respectively. The U-shaped complex housed 289 residents in 99 households. Building $\mathrm{H}$ and I collapsed on A and G, and the structure of the rest of the five buildings was distorted. Table 1 shows the death toll in each building of Weiguan-Jinlong complex. The rescue mission was an arduous challenge as heavy machinery was called for to assist rescuers. However, we completed the mission despite all the odds. Four sector command posts on four wings constituted the command system, and numerous rescuers worked on shift around the clock. The mission was split into three phases. Rapid search and rescue was the primary task in phase one. In phase two, life detectors and search dogs were utilized to position survivors. And in the last phase, heavy machinery was deployed for demolition and deep search. Based on the faith of "persist to the end and never give up," every member in every sector of the Tainan City Government understood people's suffering and shared their compassion throughout the mission.

Intending to elucidate how this earthquake with epicenter in Kaohsiung city caused serious casualties in Tainan city, this study employs TELES, Taiwan Earthquake Loss Estimation System, to explore the latent mechanism [1] [2] [3] [4].

\subsection{Objectives and Goals}

1) To illustrate the significance of how to perform anti-earthquake practices and how to coordinate the support from different cities and counties by studying 0206 earthquake in Tainan.

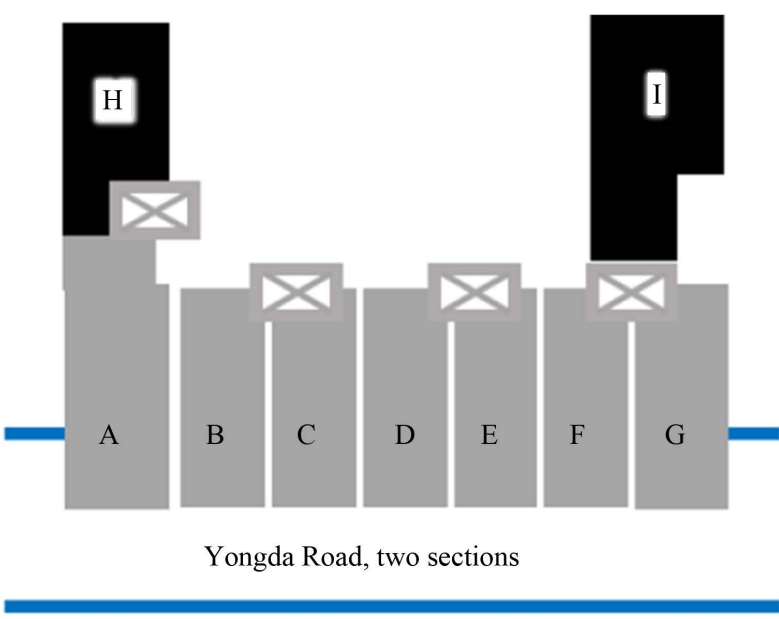

Figure 1. The schematic diagram of Weiguan Jinlong building. 
Table 1. Death toll in each building of Weiguan-Jinlong complex.

\begin{tabular}{|c|c|c|c|c|c|c|c|c|c|}
\hline & A & B & $\mathrm{C}$ & $\mathrm{D}$ & E & $\mathrm{F}$ & G & $\mathrm{H}$ & I \\
\hline Feb. 6 & 1 & 1 & 1 & & & 1 & & & \\
\hline Feb. 7 & 5 & 4 & & 3 & 3 & & 3 & 1 & \\
\hline Feb. 8 & & 1 & & & & 1 & 2 & & \\
\hline Feb. 9 & & & & & & 2 & 1 & & \\
\hline Feb. 10 & & & & 1 & & 1 & 4 & 3 & \\
\hline Feb. 11 & & 3 & & 1 & 1 & & 10 & 2 & \\
\hline Feb. 12 & 11 & 2 & & & 3 & 5 & 11 & & \\
\hline Feb. 13 & 7 & 1 & & & & & 8 & & \\
\hline Sub Total & 24 & 12 & 1 & 5 & 7 & 10 & 39 & 6 & 0 \\
\hline Total & & 115 (in & $\mathrm{ng} \mathrm{t}$. & ssing & en L & IAN & founc & eb. & \\
\hline
\end{tabular}

2) To polish the policy of disaster management to deal with the possible large-scale earthquake in Taiwan in the future.

3) To elucidate the reasons why Tainan city suffered the most serious disaster while the strongest magnitude occurred in Meinong Dist. in Kaohsiung city.

\section{Methods}

In this study, all the data are collected during the 0206 earthquake in Tainan. The data are analyzed after thorough literature review, complete present investigation and interview with experts. Next, the TELES is employed to quantify various rescue resources and establish function of different variables. TELES is developed by Taiwan National Center for Research on Earthquake Engineering, aiming to analyze the loss of earthquake disasters that may caused by the faults. Currently, TELES can be applied to estimate the intensity of ground vibrations modules. This research encompasses the earthquake source parameters and fault conditions, involving epicenter location, hypocenter depth, time of occurrence, the types of fault, cracking direction, fault inclination, length and width. Furthermore, the outcomes of TELES simulation are referred to estimate the costeffectiveness of disaster rescue and to develop rescue plan in the early stage of disaster rescue. In addition, the outcomes of TELES will facilitate how to coordinate the resources from multilevel: central government, cities and districts in the first three key days.

1) TELES application-the estimating model of trying to lives the estimating model I Number of people trapped in the wooden building ${ }^{\star} 0.226 / 4=$ number of rescue teams required.

2) Number of people trapped in the non-wooden building ${ }^{*} 0.226 / 4=$ number of rescue teams required.

Total number of rescue teams required $=(1)+(2)$.

(a) The estimating model II

The average possibility of rescuing 2 people by 1 rescue team in one day, 
within 4 days after the occurring of the earthquake.

Number of people trapped in the building ${ }^{\star} 0.226 / 2=$ number of rescue teams required.

(b) The estimating model III

The average possibility of rescuing 1.5 people by 1 rescue team in one day, within 25 days after the occurring of the earthquake.

Number of people trapped in the building ${ }^{\star} 0.226 / 1.5=$ number of rescue teams required.

In TELES system, PGA and PGV are used as the parameters in the modules, simulating the magnitude of the earth shake caused by the earthquake, to estimate the extent of damage on the structures of buildings [5] [6].

\section{Results}

Figure 2 and Figure 3 reveal that, Meinonng Dist, where the epicenter located with the strongest magnitude of PGA, had not suffered damaged building. In contrast, Yongkang Dist, with middle-leveled PGA, has suffered the most seriously damaged buildings. After the thorough investigation and exploration, experts unanimously approve that the culprits of catastrophic collapse of buildings in Yongkang Dist are the bad design and faulty construction. Therefore, following suggestions and recommendations are made to enhance the rescue miss on when large-scaled disaster occurs.

In this rescue mission, the Tainan City Government rapidly set up forward command posts to effectively manage and control all the available domestic rescue force, gears, equipment, as well as related information, for the incident commander to use. Another key to the success of this rescue mission is the initiation of "Operation Demolition Shear, which enabled us to speed up digging and send rescuers into the building early on in order to save the trapped people as quickly as possible.

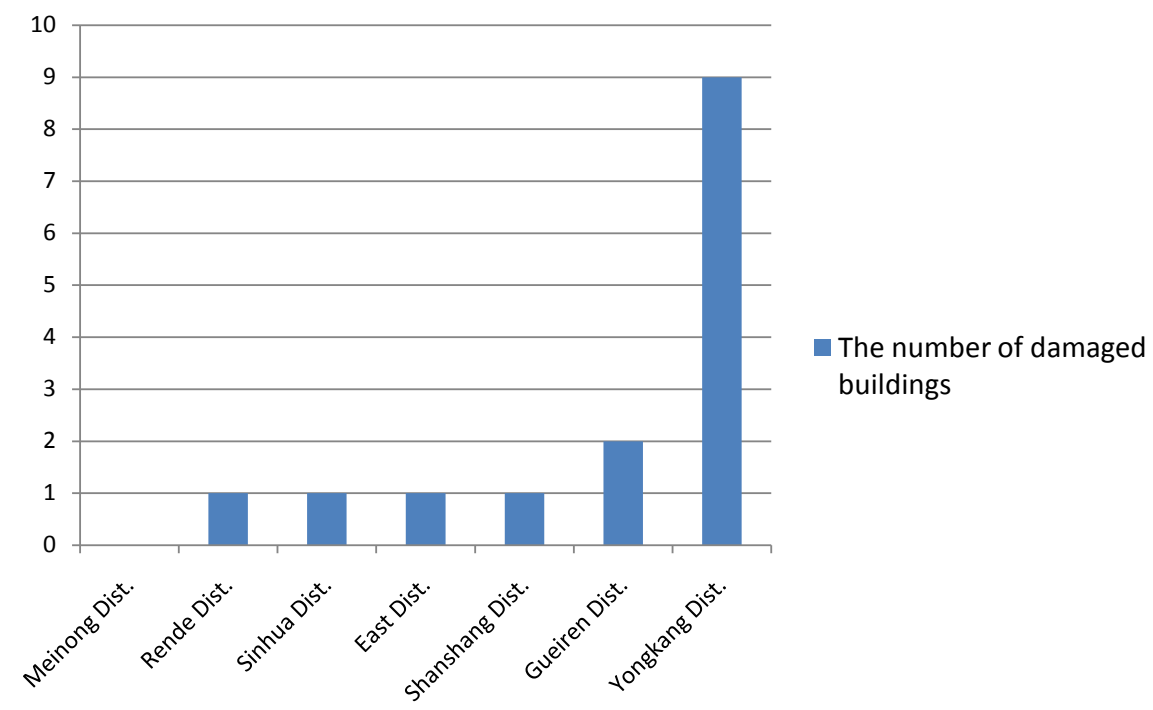

Figure 2. The comparison of the number of damaged buildings. 


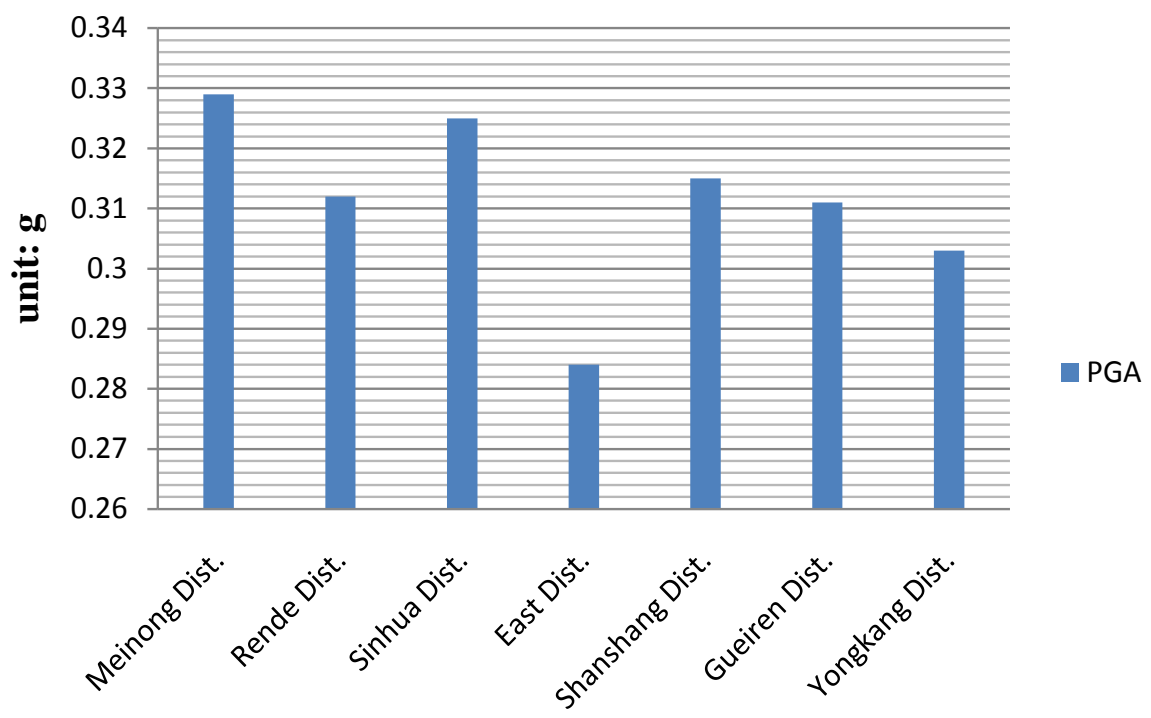

Figure 3. The comparison of the magnitude of PGA.

1) When rescuers are searching and cutting inside a building, it is advised to choose medium and small equipment, and send specialists to the disaster zone to assist rescuers if necessary.

Large handheld pneumatic or hydraulic equipment is either hard to manipulate or ineffective due to its relative large size and weight in a distorted and narrow space. Rescuers should detect signals of life in the preliminary phase of a rescue mission by cutting a small hole for life detectors to penetrate. Thus, medium or small electric equipment such as reciprocating saws, circular saws, or grinders will be more convenient. They are compact and handy, and will work even more effectively when a high quality cutting blade or grinding wheel is used. Once a trapped person is found, large equipment like an electric hammer will be used, and efficiency will be enhanced with the assistance of specialists (drillers) if conditions permit.

Also, we advise that a contact list of structural engineers should be created for rescuing assistance in a collapsed building like those in this case. The structural engineers engaged in this mission contributed a lot. They timely advised the rescuers on how to cut or break the buildings in an efficient and safe manner.

2) Signs of life should be cross-verified when using life detectors.

The noise in the scene hinders a life detector's ability to detect weak signals from life. Even if the scene is ideally silent, signals from life may be discontinuous if the trapped person's consciousness is unstable due to physical/psychological trauma or hypoxia. Signals from the trapped people may become inaudible or indistinguishable when they are interfered by the noise at the scene. Unless the signals are regular, such as knocking three times in respond to a rescuer's request, and with the readings of a life detector, signals from life may not be easy to identify. Some signals may be noises from other sources.

An example of that is the site F14. The readings from a sonar life detector 
were insufficient to determine if a survivor was trapped inside or not. A radar life detector was then used to confirm. A through-wall radar life detector emits conical ultra-high frequency waves to rubble and then analyzes the signals bounced back to determine if inside trapped a survivor or not. The readings are more reliable. If a rescue dog is paired to cross-verify the signals, the accuracy will rise. For increasing rescue efficiency in a collapsed building, we suggest that more radar life detectors be purchased.

3) Radio channels should be properly allocated for support agencies and organizations.

The Fire Bureau has separated its radio channels for years. Available channels are Channel No. 2 for island-wide communication, and Channel No. 30, 31, 32, and 36 for Tainan City only. The separation system worked well in this rescue mission. However, members of support agencies and organizations from other counties/cities and civil rescue teams could not use those channels. When radio channels were separated, support members could not reach their sector commander with radio.

Below are our solutions. First, every support agency or organization designates a liaison officer to contact their sector commander in person. When a survivor is pulled out or if there is any request for support, the liaison will be sent to contact the sector commander in person under the leader's command. Secondly, the Tainan City Government Fire Bureau provides the leader of every support agency or organization with a locally used radio and switches it to a specific channel. All the leaders will be able to contact their sector commander at any time. Thirdly, when support members are in action, the Tainan City Government Fire Bureau will designate one of its staffer to follow them, and the staffer will be in charge of contacting the sector commander.

4) A sector command post should be set up on every wing for rescue members to assemble and be under control, and meetings should be held on a regular basis.

To complete a large scale rescue mission, it takes more than just a commander to integrate information and assign tasks. Sector command posts have to be set up in order to assign tasks more specifically. The commander of every sector command post should keep a list of support members, assemble equipment, and assign operation area and time in order to integrate the rescue resources in the sector.

Besides, a spacious area has to be designated as a rendezvous for support members to rest and be under control. A rendezvous not only allows support members to have a better place to rest and dine, it also enables sector commanders to contact support agencies and organizations more easily, so the numbers of members in and out of the disaster zone will be clear. Plus, it serves as a convenient venue for regular meetings to be held. Efficiency will be achieved when leaders of all support agencies and organizations can discuss issues regarding current situations, needed support, teamwork approaches, task transfers and so on. 


\section{References}

[1] Iwasaki, T., Arakawa, T. and Tokida, K. (1982) Simplified Procedures for Assessing Soil Liquefaction During Earthquakes. Soil Dynamics and Earthquake Engineering Conference, Southampton, 925-939.

[2] Omori, F. (1907) Earthquake of the Chiayi Area, Taiwan, 1906. Introduction of Earthquake, 103-147. (In Japanese)

[3] Omori, F. (1907) Preliminary Note on the Formosa Earthquake of March 17, 1906. Bull. Imp. Earthquake Investigation Committee, 1, 53-69.

[4] Tsai, Y.B. (1986) A Study of Disastrous Earthquakes in Taiwan, 1683-1895. Bulletin of the Institute of Earth Sciences, Academia Sinica, 5, 1-44.

[5] Wells, D.L. and Coppersmith, K.J. (1994) New Empirical Relationships among Magnitude, Rupture Length, Rupture Width, Rupture Area, and Surface Displacement. Bulletin of the Seismological Society of America, 84, 974-1002.

[6] Wu, Y.M., Shin, T.C. and Chang, C.H. (2001) Near Real-Time Mapping of Peak Ground Acceleration and Peak Ground Velocity Following a Strong Earthquake. Bulletin of Seismological Society of America, 91, 1218-1228.

https://doi.org/10.1785/0120000734 